\title{
Patrones de crecimiento estatural y maxilofaciales en niños de 6 a 12 años de la ciudad de Cuenca
}

\section{Statural growth patterns and maxillofacial growth in children from 6 to 12 years of age from the city of Cuenca}

\author{
Edison Gustavo Moyano Brito*1,2, Nube Johanna Pacurucu Ávila ${ }^{1,2}$, Noren Enrique Villalobos Inciarte ${ }^{2}$, Roberto \\ Antonio García López ${ }^{2}$ \\ ${ }^{1}$ Universidad Católica de Cuenca, Ecuador \\ ${ }^{2}$ Universidad del Zulia, Venezuela \\ *emoyanob@ucacue.edu.ec
}

DOI: https://doi.org/10.26871/killkanasalud.v4i4.752

\begin{abstract}
Resumen
Objetivo: Establecer patrones de crecimiento estatural y maxilofaciales referenciales en niños de 6 a 12 años en la ciudad de Cuenca. Materiales y métodos: Estudio cuantitativo correlacional, la muestra se conformó por 1750 niños de 6 a 12 años seleccionados de manera probabilística y estratificada por edades y sexo. Los escolares de esta investigación pertenecían a las diferentes escuelas de la ciudad de Cuenca, a quienes se les tomó las medidas antropométricas como estatura, altura facial inferior y profundidad del maxilar, para el proceso de recopilación de información se utilizaron un estadiómetro y calibradores cuyos datos se registraron mediante una ficha de recolección de datos elaborado por los autores y validado a criterio de expertos. La altura facial inferior y profundidad del maxilar fueron utilizadas para desarrollar una distribución percentilar: p15, p50, p85 relacionadas a la talla del escolar, para el análisis de la talla se desarrolló la distribución mediante los valores de la media aritmética y desviación estándar. Resultados: Se estableció las estaturas promedio en niños por edad y sexo, los valores se resumieron en cinco categorías que pudieran ser utilizadas como medidas referenciales para la talla en infantes de 6 a 12 años. Las variables altura facial inferior y profundidad del maxilar se obtuvieron valores percentilares (p15, p50, p85) a partir de la estatura de los infantes obteniendo rangos de $4,4 \mathrm{~cm}$ a $7 \mathrm{~cm}$ para la altura facial inferior y de $9,1 \mathrm{~cm}$ a $12,2 \mathrm{~cm}$ para la profundidad del maxilar. Conclusión: Las medidas referenciales de la estatura en los niños obtenidas en el presente estudio varían de los valores establecidos por la Organización Mundial de la Salud (OMS) siendo la estatura de los infantes de la ciudad de Cuenca $3 \mathrm{~cm}$ por debajo del percentil 50 de la OMS. Los escolares de la ciudad de Cuenca podrían utilizar los patrones de crecimiento maxilofaciales elaboradas a partir de la estatura con la finalidad de monitorizar su adecuado crecimiento.
\end{abstract}

Palabras clave: crecimiento, niño, estatura, maxilar.

\begin{abstract}
Objective: To establish reference maxillofacial and stature growth patterns in children aged 6 to 12 years in the city of Cuenca. Materials and methods: Quantitative correlational study, the sample consisted of 1750 children from 6 to 12 years old, selected in a probabilistic way and stratified by age and sex. The schoolchildren belonged to the different schools in the city of Cuenca, from whom anthropometric measurements such as height, lower facial height and maxillary depth were taken; a height rod and calipers were used to collect the information, the data of which were recorded by means of a data collection sheet prepared by the authors and validated at the discretion of experts. The lower facial height and depth of the maxilla were used to develop a percentile distribution: p15, p50, p85 related to the height of the schoolchild, for the height analysis the distribution was developed through the values of the mean and standard deviation. Results: The average sizes in children were established by age and gender, the values were summarized in five categories that could be used as referential measurements for the height in infants from 6 to 12 years old. The variables lower facial height and maxillary depth were obtained percentile values $(p 15, p 50, p 85)$ from the height of the infants, obtaining ranges from $4.4 \mathrm{~cm}$ to $7 \mathrm{~cm}$ for the lower facial height and from $9.1 \mathrm{~cm}$ to $12.2 \mathrm{~cm}$ for maxillary depth. Conclusion: The reference measures of height in children obtained in the present study vary from the values established by the World Health Organization (WHO), with the height of infants in the city of Cuenca being $3 \mathrm{~cm}$ below the 50th percentile of the WHO. Schoolchildren in the city of Cuenca could use the maxillofacial growth patterns elaborated from the height in order to monitor their adequate growth.
\end{abstract}

Keywords: growth, child, height, maxilla. 


\section{Introducción}

En la actualidad, la literatura de referencia sobre la teoría del crecimiento se considera una de las herramientas más utilizadas y valiosas en el diagnóstico clínico, para evaluar y comprender el bienestar de las personas que integran la comunidad en la que viven, en este sentido, los niños son el grupo de edad más importante en la evaluación y ponderación del crecimiento.?? Por lo que el uso de parámetros de crecimiento inapropiados para predecir la salud de los niños puede llevar a conclusiones erróneas sobre el crecimiento, estado nutricional y diagnóstico precoz de enfermedades relacionadas al desarrollo.?,?

El crecimiento es el mejor indicador mundial de la salud infantil, en este contexto el crecimiento general y maxilofacial en la primera infancia muestra enormes diferencias en tamaño, forma, función y fuerza. Sin embargo, se sabe poco sobre la investigación del crecimiento maxilofacial en niños de la segunda infancia. La investigación antropológica ha utilizado la antropometría y planos de regiones maxilofaciales para analizar el crecimiento e inferir la categoría biológica a la que pertenecen durante décadas. En la población humana, el interés por la morfología facial ha superado la definición de categorías biológicas, investigaciones realizadas por antropólogos, médicos, dentistas, pediatras y cirujanos lo demuestran porque se trata de evaluar huesos y tejidos blandos (músculos, Grasa y piel) para determinar la singularidad individual.?

El crecimiento de las diferentes áreas que componen el complejo maxilofacial, la morfología facial cambia gradualmente. Esto significa que la investigación sobre los cambios en estos componentes y sus expresiones es de gran interés para disciplinas como la pediatría, la odontología y la antropología forense. En los casos forenses y en odontología y odontología pediátrica, comprender el proceso de desarrollo y maduración facial en las diferentes etapas del rostro puede ayudar a mejorar la precisión del proceso de reconocimiento y reconstrucción facial. ${ }^{?}$ Los cambios maxilares aparecen a nivel lateral. Este es un problema común en ortodoncia. El resultado es una oclusión posterior o mordida en tijera. Suelen aparecer unilaterales, bilaterales o únicos. Debido a estos cambios, es necesario dar un diagnóstico correcto y tratamiento oportuno.??

Como antecedente de la investigación, podemos considerar la encuesta longitudinal realizada por González. En 2013,? realizó una caracterización de crecimiento general y craneofacial mediante la elaboración de patrones en 265 niños y niñas colombianos de 0 a 3 años residentes en Bogotá. En este estudio, los autores sugirieron replicar el método en distintos sectores del país y extender la muestra a cinco años.

Estudios realizados en poblaciones tempranas de estudios craneofaciales longitudinales, como los realizados por Bookstein? y Moss, ' quienes establecieron el comportamiento de crecimiento de la cúpula y la base del cráneo después del estudio. Otros estudios realizados por Farkas, Hellman, ${ }^{?}$ Enlow ${ }^{?}$ y Scott ${ }^{?}$ involucran el aumento de los pa- trones de crecimiento facial y los tres planos anatómicos del espacio, mediante estudios longitudinales y transversales para obtener la anchura, profundidad y altura, los autores coinciden que el crecimiento en las tres dimensiones tiene cierta proporcionalidad en el tiempo.

En la actualidad la investigación longitudinal, debido al alto costo, el tamaño de las muestras requeridas, la pérdida de algunas muestras durante el proceso de investigación, la falta de individuos, las limitaciones ocasionadas por el reemplazo o pérdida de interés en la investigación ha perdido espacio en los estudios antropométricos y ha sido remplazada por investigaciones transversales, en este sentido la investigación maxilofacial más relevante es la realizada por Farkas quien describió los cambios en áreas específicas en el rostro, la cabeza y sus relaciones dentro y entre áreas estaturales y maxilofaciales.?

La importancia de los estudios de crecimiento es el desarrollo de estándares o métodos antropométricos que caractericen a una población, Ecuador muestra una gran diversidad demográfica, cultural y étnica en su región, lo que incide en sus patrones de crecimiento, por lo que el presente estudio tiene por objetivo establecer patrones de crecimiento estatural y maxilofaciales referenciales en infantes de 6 a 12 años en la ciudad de Cuenca.

\section{Materiales y métodos}

El presente estudio tiene un enfoque cuantitativo de alcance correlacional y de tipo transversal, mediante la cual se investigaron a infantes de 6 a 12 años de edad pertenecientes a la ciudad de Cuenca. Los escolares investigados se encontraban cursando la educación básica. La población de investigación estuvo constituido por 24384 alumnos de 6 a 12 años de edad de las diferentes escuelas urbanas de la ciudad de Cuenca, para el cálculo del tamaño de la muestra se utilizó un intervalo de confianza del $95 \%$ y un margen de error del $3 \%$ además se consideró una proporción de 0,50. Obteniendo una muestra óptima de 1750 infantes, siendo 875 de sexo masculino y de igual forma 875 de sexo femenino, el muestreo fue probabilístico por estratos, distribuidos en 7 grupos: G1(72-83 meses), G2(84-95 meses), G3(96-107 meses), G4(108-119 meses),G5(120131 meses), G6(132-143 meses) y G7(144-155 meses) con un total de 250 infantes para cada uno de los estratos respectivamente.

En el instante de la investigación de aplicar las mediciones antropométricas se confirmó los registros de nacimiento, esto se verificó mediante los registros de cada una de las instituciones educativas, para el proceso de recopilación de información se aplicaron un estadiómetro y calibradores cuyos datos se registraron mediante una ficha de recolección de información realizado por los autores y validado a criterio de expertos. La altura facial inferior y profundidad del maxilar fueron utilizadas para la construcción de una distribución percentilar: p15, p50, p85 relacionadas a la estatura del escolar, para el análisis de la estatura se desarrolló 
la distribución mediante los valores de la media aritmética y desviación estándar.

Para la medición de las variables de tipo antropométricas se siguieron los protocolos estandarizados a nivel internacional, mediante las normas establecidas por «International Working Group of Kineanthropometry» investigado por Ross y Marfell-Jones. ' Las variables de estudio se describen a continuación:

- $\quad$ Estatura (cm): Se realizó la evaluación de la estatura descalzo, el infante ubicado en el plano de Frankfurt, mediante el uso de un estadiómetro marca Seca graduada con una escala en milímetros.

- Altura facial inferior (cm): Se evaluó la distancia comprendida entre la base de la nariz (Sn) y la parte más inferior del mentón (Gn), ubicado el infante en el plano de Frankfurt, utilizando un calibrador digital pie de Rey marca Vernier.

- $\quad$ Profundidad del maxilar (cm): Se evaluó la distancia comprendida entre la base de la nariz ( $\mathrm{Sn}$ ) y la parte anterior del conducto auditivo externo, ubicado el infante en el plano de Frankfurt, utilizando un calibrador digital pie de Rey marca Vernier.

Para garantizar una mejor calidad y evitar sesgos durante los registros antropométricos, se realizó una medición doble cada 10 infantes en todas las variables de estudio de esta manera se puede conseguir un coeficiente de reproductibilidad altamente confiable.

Finalmente, para el análisis estadístico se calculó distribución percentilar mediante la media aritmética y desviación estándar de las variables cuantitativas como la estatura, altura facial inferior y profundidad del maxilar y en las variables cualitativas se analizarán mediante tablas de frecuencias mediante el programa estadístico SPSS.

\section{Resultados}

De los 1750 infantes evaluados, $875(50 \%)$ fueron de sexo masculino y $875(50 \%)$ del sexo femenino. La totalidad de la muestra se distribuyeron en 7 grupos con un total de 250 infantes para cada uno de los estratos respectivamente (Tabla 1)

Tabla 1: Caracterización de la población estudiada

\begin{tabular}{lcc}
\hline & Frecuencia & Porcentaje $\%$ \\
\hline Edad & & \\
\hline 6 años & 250 & $14,28 \%$ \\
7 años & 250 & $14,28 \%$ \\
8 años & 250 & $14,28 \%$ \\
9 años & 250 & $14,28 \%$ \\
10 años & 250 & $14,28 \%$ \\
11 años & 250 & $14,28 \%$ \\
Totan & 250 & $14,28 \%$ \\
\hline Sexo & 1750 & $100 \%$ \\
\hline Masculino & & \\
Femenino & 875 & $50 \%$ \\
Total & 875 & $50 \%$ \\
\hline
\end{tabular}

Para la construcción de la distribución de la estatura de acuerdo a la edad de los infantes se utilizó la técnica LMS que es un modelo estructural no matemático que permite ajustar información antropométrica transversal y obtener estándares de percentiles normalizados. La técnica indicada asume que, para cada estrato de edad, los valores antropométricos pueden calibrarse de manera que se ajuste a un tipo de distribución normal y de esta manera transformarlos de una manera adecuada, teniendo presente el nivel de asimetría (L), el valor de la tendencia central (M) y el valor de dispersión (S). La tabla 2 describe los valores de los percentiles p3, p15, p50, p85 y p97 ajustados al método LMS de las estaturas en los infantes de ambos sexos.

Tabla 2: Valores percentiles para la estatura en niños de 6 a 12 años de ambos sexos de acuerdo a la media aritmética y desviación estándar

\begin{tabular}{|c|c|c|c|c|c|}
\hline $\begin{array}{l}\text { EDAD EN } \\
\text { AÑOS }\end{array}$ & $\begin{array}{l}\text { P3 BAJA TALLA } \\
\text { SEVERA }(\mathrm{cm})\end{array}$ & $\begin{array}{l}\text { P15 BAJA } \\
\text { TALLA }(\mathrm{cm})\end{array}$ & $\begin{array}{l}\text { P50 NORMAL } \\
(\mathrm{cm})\end{array}$ & $\begin{array}{l}\text { P85 TALLA } \\
\text { ALTA }(\mathrm{cm})\end{array}$ & $\begin{array}{l}\text { P97 TALLA } \\
\text { MUY ALTA } \\
(\mathrm{cm})\end{array}$ \\
\hline \multicolumn{6}{|c|}{ Niños } \\
\hline 6 & 97,9 & 102,7 & 112,3 & 121,9 & 126,7 \\
\hline 7 & 103,4 & 108,2 & 117,8 & 127,4 & 132,2 \\
\hline 8 & 109,7 & 114.5 & 124,1 & 133,7 & 138,5 \\
\hline 9 & 115,0 & 119,8 & 129,4 & 139,0 & 143,1 \\
\hline 10 & 121,3 & 126.1 & 135,7 & 145,3 & 150,1 \\
\hline 11 & 127,1 & 131,9 & 141,5 & 151,1 & 155,9 \\
\hline 12 & 132,8 & 137,6 & 147,2 & 156,8 & 161,6 \\
\hline \multicolumn{6}{|c|}{ Niñas } \\
\hline 6 & 95,8 & 100,6 & 110,2 & 119,8 & 124,6 \\
\hline 7 & 101,2 & 106,0 & 115,6 & 125,2 & 130,0 \\
\hline 8 & 107,9 & 112,7 & 122,3 & 131,9 & 136,7 \\
\hline 9 & 113,3 & 118,1 & 127,7 & 137,3 & 142,1 \\
\hline 10 & 119,4 & 124,2 & 133,8 & 143,4 & 148,2 \\
\hline 11 & 125,2 & 130,0 & 139,6 & 159,2 & 154,0 \\
\hline 12 & 130,9 & 135,7 & 145,3 & 154,9 & 159,7 \\
\hline
\end{tabular}


Se puede observar una relación lineal entre las variables estatura y edad en donde existe un aumento no exponencial de la estatura con los diferentes grupos de edad según la comparación de la mediana con los valores percentilares de cada uno de los diagramas de caja y línea expresados en la figura 1.

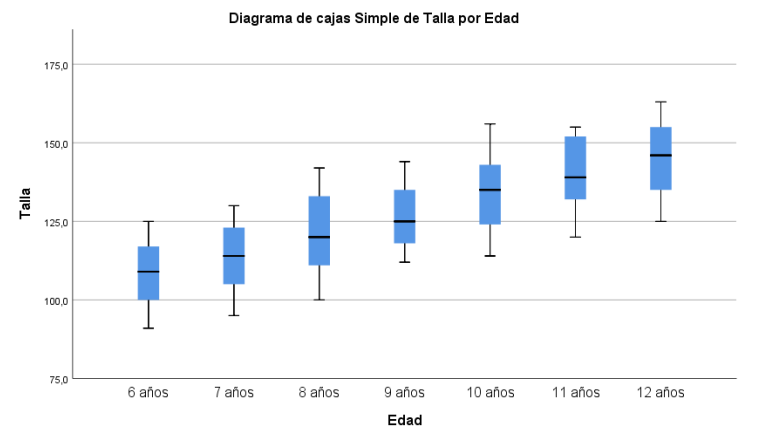

Fig. 1: Diagrama de caja y línea por grupo de edad en relación a la distribución de la estatura en los infantes

Las tablas 3 y 4 presentan la distribución percentilar de la profundidad maxilar y altura facial inferior respectivamente de acuerdo a los rangos de estatura de los infantes de 6 a 12 años de edad. Esta distribución va desde el percentil 5 hasta el percentil 95 y podría ser utilizada como referencia para representar la variabilidad de estos parámetros de acuerdo a la estatura de los infantes.

Los valores entre el percentil 5 y percentil 95 son considerados como límites de normalidad de acuerdo a la probabilidad relativa en la Campana de Gauss. Estos valores percentilares desde la perspectiva de la estadística son utilizados en estudios antropométricos, debido a que nos permiten identificar el estado de riesgo de grupos poblacionales.

Tabla 3: Distribución percentilar para la profundidad del maxilar de acuerdo a la estatura en infantes de 6 a 12 años

\begin{tabular}{lccc}
\hline \multirow{2}{*}{ Rango de Estatura $(\mathrm{cm})$} & \multicolumn{3}{c}{ Valores de la profundidad del maxilar $(\mathrm{cm})$} \\
\cline { 2 - 4 } & Percentil 5 & Percentil 50 & Percentil 95 \\
\hline $90-110$ & 9,1 & 10,1 & 11 \\
$111-130$ & 9,6 & 10,6 & 11,5 \\
$131-150$ & 10,3 & 11,3 & 12,2 \\
\hline
\end{tabular}

Tabla 4: Distribución percentilar para la altura facial inferior de acuerdo a la estatura en infantes de 6 a 12 años

\begin{tabular}{lccc}
\hline \multirow{2}{*}{ Rango de Estatura $(\mathrm{cm})$} & \multicolumn{3}{c}{ Valores de la altura facial inferior $(\mathrm{cm})$} \\
\cline { 2 - 4 } & Percentil 5 & Percentil 50 & Percentil 95 \\
\hline $90-110$ & 4,4 & 5,1 & 5,8 \\
$111-130$ & 4,9 & 5,6 & 6,3 \\
$131-150$ & 5,6 & 6,3 & 7 \\
\hline
\end{tabular}

\section{Discusión}

La evaluación del crecimiento estatural en la mayor parte de los países se realiza mediante el uso de curvas de referencia establecidas por la Organización Mundial de la Salud? ?' sin embargo, para Tarupia et al en su estudio sobre la construcción de curvas de crecimiento expresa que utilizar como referencia las curvas de crecimiento de otro lugar es inapropiado y puede producir errores en el momento de tomar decisiones, por lo que considera importante que cada país tenga sus propios patrones referenciales de crecimiento? .

En virtud a lo expuesto el presente estudio elaboró parámetros referenciales de la estatura para infantes de 6 a 12 años de edad de la ciudad de Cuenca en donde se evidenció estaturas inferiores de aproximadamente 3 centímetros para cada uno de los rangos de edad en comparación con las estaturas referenciales establecidas por la Organización Mundial de la salud, ${ }^{?}$ de igual manera se pudo observar una diferencia entre las estaturas de los infantes de sexo masculino y femenino en todas las edades de aproximadamente 2 centímetros.

Los parámetros de estatura de este estudio al igual que los establecidos por la Organización Mundial de Salud se realizaron por medio del método LMS que es una técnica no estructural matemático que permite ajustar datos antropométricos transversales y obtener estándares de percentiles normalizados, este método establece referencias estandarizadas de crecimiento a partir de un pequeño número de parámetros interpretables biológicamente. ? Factores como las diferencias raciales o étnicas, composición corporal pueden complicar la construcción de estos patrones de crecimiento.

En Colombia en un estudio realizado por González propone el uso de patrones y las curvas referenciales de crecimiento craneofacial como parte integral en la consulta de control de crecimiento y desarrollo del infante, con el propósito de brindar un instrumento para el diagnóstico temprano de las alteraciones que pudieran presentarse, $?$ sin embargo a nivel de nuestro país y de América Latina no existen estudios relacionados a la construcción de patrones de crecimiento referenciales de la región maxilofacial, por lo tanto surge la necesidad de realizar estudios que conlleven a la construcción de referencias de crecimiento de las regiones maxilofaciales, basados en mediciones de infantes Ecuatorianos y que representen las características antropométricas de niños del Ecuador, para que puedan ser usadas en la consulta odontológica con la finalidad de hacer diagnóstico temprano en pacientes con alteraciones en el desarrollo del complejo maxilofacial.

Este estudio tomó de referencia a la altura facial inferior y la profundidad del maxilar como valores referenciales debido a que estos parámetros tras las pruebas estadísticas de Pearson presentaron una relación significativa con respecto a la estatura de los infantes, este resultado coincide con el estudio realizado por Echeverri J et al. en donde establecen que existe una correlación positiva entre el crecimiento estatural y el crecimiento facial.,?

Los valores obtenidos de la altura facial inferior y la profundidad del maxilar fueron ordenados mediante una distribución percentilar según rangos de estatura, en donde 
se tomó como referencia al percentil 5 y percentil 95 que son estos valores considerados como límites de normalidad y permiten identificar el estado de riesgo de grupos poblacionales. Actualmente no existen investigaciones en otros países sobre estos indicadores de crecimiento maxilofacial que permitan comparar los valores obtenidos en la presente investigación.

\section{Conclusiones}

Las medidas referenciales de la estatura en los niños obtenidas en el presente estudio varían de los valores establecidos por la Organización Mundial de la Salud (OMS) siendo la estatura de los infantes de la ciudad de Cuenca $3 \mathrm{~cm}$ por debajo del percentil 50 de la OMS.

Las tablas de crecimiento maxilar constituyen la primera referencia descriptiva del crecimiento maxilofacial de infantes ecuatorianos de 5 a 12 años, pudiendo usarse estos indicadores con la finalidad de monitorizar su adecuado crecimiento o como punto de partida para estudios posteriores

\section{Fuente de Financiamiento}

Este estudio es autofinanciado.

\section{Conflicto de Intereses}

No existen conflictos personales, profesionales, financieroso de otro tipo.

\section{Consentimiento Informado}

Los autores cuentan con el consentimiento informado de los pacientes para la investigación, la publicación del caso y sus imágenes.

\section{Referencias Bibliográficas}

1. Cole TJ, Green PJ. Smoothing reference centile curves: The lms method and penalized likelihood. Stat Med [Internet]. 1992 Jan 1 [cited 2021 Feb 4];11(10):1305-19. Available from: http://doi.wiley.com/10.1002/sim.4780111005

2. Métodos de valoración del estado nutricional [Internet]. [cited 2021 Feb 4]. Available from: http://scielo.isciii.es/scielo.php?script=sci_arttext\& pid=S0212-16112010000900009

3. Olsen EM, Petersen J, Skovgaard AM, Thomsen BL, Jørgensen T, Weile B. The growth pattern of 0-1-yearold Danish children, when screened by public health nurses - The Copenhagen County Child Cohort 2000. Ann Hum Biol [Internet]. 2005 May [cited 2021 Feb 4];32(3):297-315. Available from: https://pubmed.ncbi. nlm.nih.gov/16099775/

4. Witriw A. PROGRAMA DE EVALUACION NUTRICIONAL. 2019 [cited 2021 Feb 4]; undefinedundefined. Available from: https://www.mendeley.com/ catalogue/a122777c-adc4-35e6-a071-eb27f1125241/ ?utm_source=desktop\&utm_medium $=1.19 .4 \&$
utm_campaign=open_catalog\&userDocumentId= \%7B89fa628d-4f44-3aa7-896f-5c169649cbd0\%7D

5. Zemel BS, Riley EM, Stallings VA. Evaluation of methodology for nutritional assessment in children: Anthropometry, body composition, and energy expenditure [Internet]. Vol. 17, Annual Review of Nutrition. Annu Rev Nutr; 1997 [cited 2021 Feb 4]. p. 211-35. Available from: https://pubmed.ncbi.nlm.nih.gov/9240926/

6. E V, F C, S M. Computer-aided morphological analysis for maxillo-facial diagnostic: a preliminary study. J Plast Reconstr Aesthet Surg [Internet]. 2010 [cited 2021 Feb 4];63(2). Available from: https://pubmed.ncbi.nlm. nih.gov/19059819/

7. Duran P, Caballero B, de Onis M. The association between stunting and overweight in Latin American and Caribbean preschool children. Food Nutr Bull. 2006;27(4):300-5.

8. Tedaldi J, Calderón R, Mayora L, Quirós O, Farias M, Rondóm S, et al. Tratamiento de maloclusiones según el estadio de maduración carpal. 2007 [cited 2021 Feb 4];1-30. Available from: https://www.mendeley.com/catalogue/de29fc92-d79f3d8e-9238-ca9cc363357c/?utm_source=desktop\& utm_medium $=1.19 .4 \&$ utm_campaign $=$ open_catalog $\&$ userDocumentId=\%7Bf4714d74-bdcc-3fd0-a713208eb3b4bde9\%7D

9. Yaned González Bejarano L. Biometría Craneofacial en niños de 0-3 años Un estudio longitudinal-Caso Colombia. 2013.

10. Bookstein FL. The geometry of craniofacial growth invariants. Am J Orthod. 1983 Mar 1;83(3):221-34.

11. Moss ML, Salentijn L. The primary role of functional matrices in facial growth. Am J Orthod. 1969 Jun 1;55(6):566-77.

12. Marentette L. Anthropometric facial proportions in medicine. Edited by Leslie G. Farkas and Ian R. Munro, 344 pp, Charles C Thomas, Springfield, Illinois, 1987, Head Neck Surg [Internet]. 1988 Jan 1 [cited 2021 Feb 4];10(S1):S80-1. Available from: http://doi.wiley.com/10. 1002/hed.2890100718

3. Hellman M. Ossification of epiphysial cartilages in the hand. Am J Phys Anthropol [Internet]. 1928 Jan 1 [cited 2021 Feb 4];11(2):223-57. Available from: http://doi. wiley.com/10.1002/ajpa.1330110205

14. Crecimiento MaxiloFacial DONALD H. ENLOW.pdf | Piel | Cráneo [Internet]. [cited 2021 Feb 4]. Available from: https://es.scribd.com/document/405093816/ Crecimiento-MaxiloFacial-DONALD-H-ENLOW-pdf

15. Scott JH. The Growth of the Human Face. J R Soc Med [Internet]. 1954 [cited 2021 Feb 4];47(2):91-100. Available from: https://www.ncbi.nlm.nih.gov/pmc/articles/ PMC1918731/

16. Farkas LG, Posnick JC, Hreczko TM, Pron GE. Growth Patterns of the Nasolabial Region: A Morphometric Study. Cleft Palate-Craniofacial J [Internet]. 1992 Jul [cited 2021 Feb 4];29(4):318-24. Available from: https://pubmed. ncbi.nlm.nih.gov/1643060/ 
17. ROSS, WD. Kinanthropometry. Physiol Test Highperformance Athl [Internet]. 1991 [cited 2021 Feb 4]; Available from: https://ci.nii.ac.jp/naid/10015393589

18. OMS I El estado físico: uso e interpretación de la antropometría [Internet]. [cited 2021 Feb 4]. Available from: https://www.who.int/childgrowth/publications/ physical_status/es/

19. Patrones de crecimiento [Internet]. [cited 2021 Feb 4]. Available from: http://www.incap.int/sisvan/index. php/es/areas-tematicas/herramientas-operacionales-deapoyo/patrones-de-crecimiento

20. Referencias de peso, estatura e índice de masa corporal para niñas y niños ecuatorianos de 5 a 19 años de edad. Arch Argent Pediatr. 2020 Feb 1;118(2).

21. Rebato E, Rosique J, Salces I, San Martín L, Fernández JR, Vinagre A, et al. Estudio comparativo entre el PB1 y la combinación del PB1 con el LMS en una muestra transversal de Bizkaia [Internet]. [cited $2021 \mathrm{Feb} 4]$. Available from: www.didac.ehu.es/antropo

22. CRECIMIENTO Y DESARROLLO FACIAL Y GENERAL DE INDIVIDUOS DEL VALLE DEL ABURRA I CES Odontología [Internet]. [cited 2021 Feb 4]. Available from: https://revistas.ces.edu.co/index.php/ odontologia/article/view/1771

23. Barrera-Dussán N, Ramos-Castañeda JA. Prevalencia de malnutrición en menores de 5 años. Comparación entre parámetros OMS y su adaptación a Colombia. Univ y Salud. 2019 Dec 30;22(1):92-6.

Recibido: 9 de octubre de 2020

Aceptado: 25 de octubre de 2020

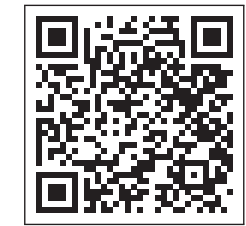

